\title{
Generation of siRNAs by T-DNA Sequences Does Not Require Active Transcription or Homology to Sequences in the Plant
}

\author{
Tomas Canto, Fabrizio Cillo, and Peter Palukaitis \\ Scottish Crop Research Institute, Invergowrie, Dundee DD2 5DA, Scotland, U. K.
}

Submitted 15 May 2002. Accepted 25 July 2002.

\begin{abstract}
Delivery into plants of T-DNAs containing promoter, terminator, or coding sequences generated small interfering RNAs (siRNAs) specific to each type of sequence. When both promoter and transcribed sequences were simultaneously present in the T-DNA, accumulation of siRNAs to transcribed sequences was favored over accumulation of siRNAs to the nontranscribed upstream promoter sequences. The generation of specific siRNA sequences occurred even in the absence of T-DNA homology to sequences in the plant. Delivery of T-DNA, with homology to the transgene limited to the nontranscribed cauliflower mosaic virus $35 \mathrm{~S}$ promoter (35SP) and the transcribed nopaline synthase transcription termination (NosT)signal sequences, into transgenic plants expressing the green fluorescent protein (GFP), generated siRNAs in infiltrated tissues to both 35SP (35SsiRNAs) and NosT (NosTsiRNAs), but not to the GFP sequence (GFPsiRNAs). In infiltrated tissues, the 35SsiRNAs failed to trigger the transcriptional silencing of the transgene, accumulation of 35SsiRNAs could be prevented by the potyviral HC-Pro, and the NosTsiRNAs required an initial amplification to trigger efficient transgene silencing, which is mediated by transcripts from the exogenous T-DNA, and not from the transgene. In upper leaves, silencing correlated with the presence of GFPsiRNAs and the absence of 35SsiRNAs, confirming that its spread was posttranscriptionally mediated by the transgene mRNA.
\end{abstract}

RNA-mediated processes capable of suppressing the expression of specific genes have been described in several types of organisms. This phenomenon, generically referred to as gene silencing, is called RNA interference (RNAi) in animals (Grishok et al. 2001; Tuschl 2001) and quelling in fungi (Cogoni and Macino 1999). In plants, gene silencing usually is called posttranscriptional gene silencing (PTGS) when it involves degradation of transcribed RNA and transcriptional gene silencing (TGS), when it is induced by promoter methylation (Vaucheret and Fagard 2001). The mechanisms underlying gene silencing in these different organisms share a number of similarities. In all cases, silencing depends on sequence homology. At some point it requires the presence of doublestranded (ds)RNAs as triggers or intermediates from which double-sided, small interfering RNAs (siRNAs) originate. The siRNAs lead the silencing machinery to the targeted sequences (Dalmay et al. 2000; Elbashir et al. 2001; Hamilton and

Corresponding author: P. Palukaitis; Telephone: +44(0)1382 562 731; Fax: +44(0)1382 562 426. E-mail: ppaluk@ scri.sari.ac.uk
Baulcombe 1999; Zamore et al. 2000). The availability of genetic tools and cell extracts in the Arabidopsis and Drosophila models has allowed the dissection of the pathways of gene silencing, as well as both the identification of some of the genes and cofactors involved and the determination of their properties (Bernstein et al. 2001; Dalmay et al. 2000; Fagard et al. 2000; Hammond et al. 2000; Lipardi et al. 2001; Mourrain et al. 2000; Nykänen et al. 2001).

It seems now that gene silencing as a mechanism of defense against foreign genes in plants (Brigneti et al. 1998; Ratcliff et al. 1997; Voinnet 2001; Voinnet et al. 1999) may be using pathways that, in other organisms, have been involved in the translational regulation of gene expression. These latter pathways generate small temporary RNAs (stRNAs), and newly discovered populations of microRNAs (miRNAs), which occur in several organisms. stRNAs are involved in translational regulation of gene expression and also originate from the activity of the Dicer ribonuclease or homologues. However, they are single-stranded (ss) and there is no evidence that stRNAs induce mRNA degradation (Grishok et al. 2001; LagosQuintana et al. 2001; Lau et al. 2001; Lee and Ambros 2001; Wightman et al. 1993).

The induction of PTGS involves not only the suppression of transgene mRNA levels, via siRNAs, but also correlates with de novo methylation of the transgene DNA corresponding to the transcribed sequences (Jones et al. 1998; Pélissier et al. 1999; Wassenegger et al. 1994). However, this is not the case so far for endogenous genes (Jones et al. 1999; Thomas et al. 2001; Vaistij et al. 2002). On the other hand, transgene promoter sequences expressed from an RNA virus vector or, in some cases, transcribed from another upstream promoter as inverted repeats, induced methylation of the corresponding DNA promoter sequences due to proposed RNA-DNA interactions (Jones et al. 1999; Mette et al. 1999; Wassenegger et al. 1994). In the latter case, promoter methylation was triggered by dsRNA with sequences identical to promoter sequences (Mette et al. 2000).

Delivery of DNA into the plant cells by agroinfiltration has been used for the transient expression of genes in plants and for the induction of systemic silencing of transgenes (Johansen and Carrington 2001; Voinnet 2001). This technique takes advantage of the ability of Agrobacterium tumefaciens to transfer a linear ssDNA (the transfer or T-DNA) into the plant nucleus (Tinland et al. 1994). Delivery of such ectopic DNA with sequence homology to a transgene can trigger the silencing of the transgene by mechanisms not fully understood. Silencing requires the generation of siRNAs that are part of the mRNA degradation machinery (Lipardi et al. 2001). In the case of promoterless, and therefore nontranscribed, ectopic DNA sharing 

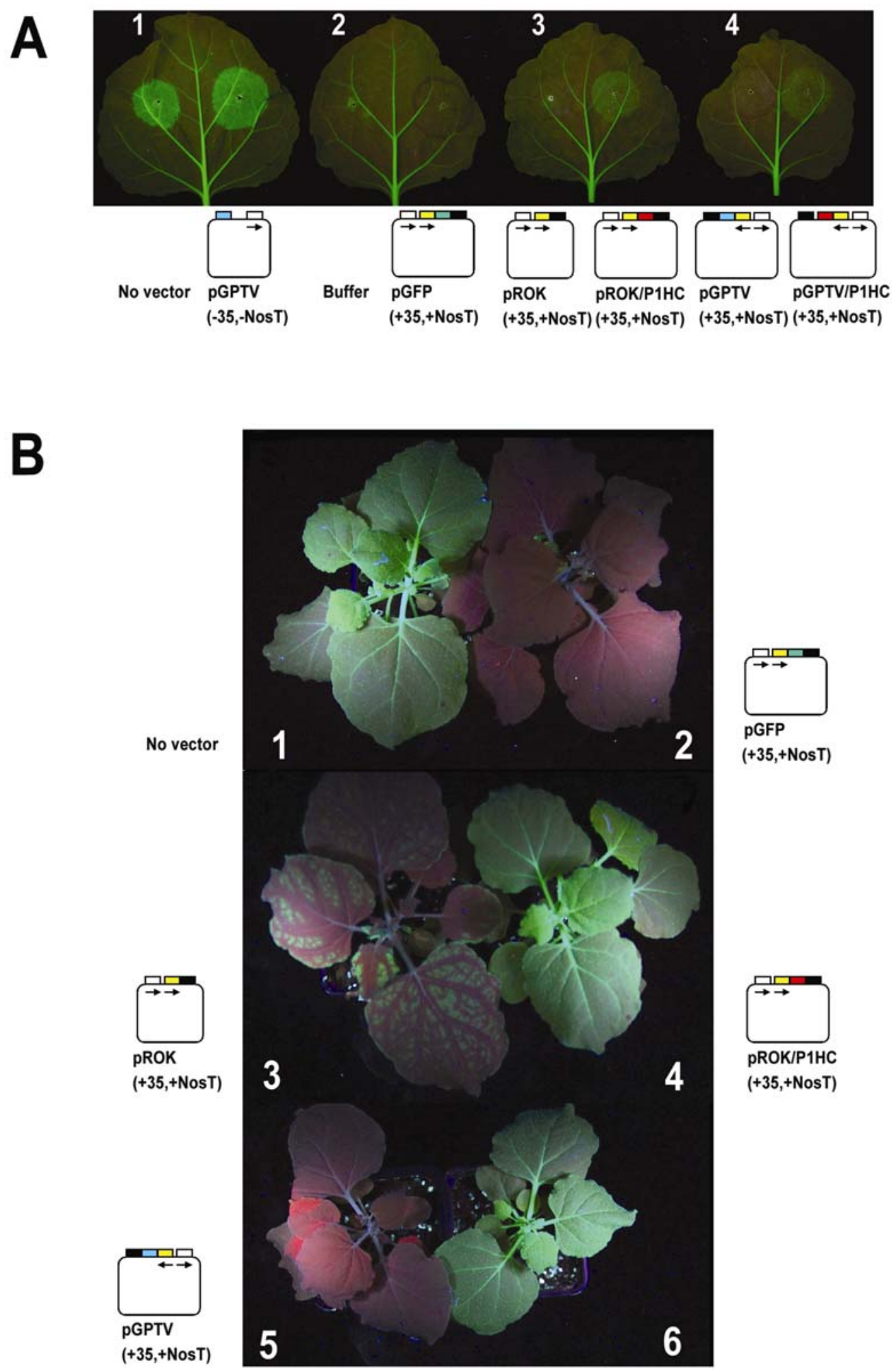

Fig. 1. A, UV detection of green fluorescent protein (GFP)-derived fluorescence at 12 days post infiltration of leaves from Nicotiana benthamiana Line $16 \mathrm{c}$ plants infiltrated with Agrobacterium tumefaciens harboring either no vector (left side of leaf 1), or with buffer alone (infiltrated area on left side of leaf 2) or

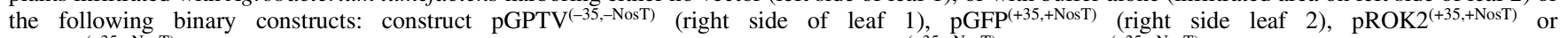

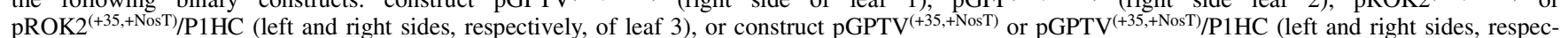
tively, of leaf 4). B, Systemic silencing of the transgene encoding the GFP makes the plants appear red under ultraviolet illumination due to chlorophyll autofluorescence. Nonsilenced plants fluoresce green. Photographs of $N$. benthamiana Line 16c plants that had been infiltrated 3 weeks before (four- to sixtrue-leaf stage) with A. tumefaciens harboring no binary vector (plant 1), construct pGFP ${ }^{(+35,+\mathrm{NosT})}$ (plant 2 ), construct pROK2 ${ }^{(+35,+\mathrm{NosT})}$ (plant 3 ), construct $\mathrm{pROK}^{(+35,+\mathrm{NosT})} / \mathrm{P} 1 \mathrm{HC}$ (plant 4), or construct $\mathrm{pGPTV}^{(+35,+\mathrm{NosT})}$ (plant 5). Plant 6 shows a noninfiltrated plant. Each constructs is schematically represented with different color-coded rectangles indicating the different sequences: 35SP, yellow; GFP, green; NosT, black; P1HCPro, red; $\beta$-glucuronidase (GUS), blue; and the cassettes NosP:nptII:NosT (for pROK2 constructs) or NosP:nptII:pAg7T (for the pGPTV constructs), open rectangles. Arrows below the promoters indicate the direction of transcription. Abbreviations: 35SP, Cauliflower mosaic virus 35S RNA promoter; P1HC or P1HCPro, Potato virus $Y$ P1/helper component protease; NosT, nopaline synthase transcription terminator signal; NosP, nopaline synthase promoter; nptII, neomycin phosphotransferase; pAg7T, gene 7 poly(A) transcription termination signal. 
sequence homology to a transgene coding region, transgene silencing was proposed to be triggered by interactions between the ectopic DNA and the transgene (Voinnet et al. 1998). On the other hand, it was concluded that promoter sequences in the ectopic DNA not transcribed into RNA played no role in transgene silencing (Schöb et al. 1997; Voinnet et al. 1998). Thus, the role of the noncoding sequences flanking a transgene in the silencing of a transgene triggered by ectopic DNA is not clear. We have examined this here and show that T-DNA sequences corresponding to transgene noncoding regions, agroinfiltrated as binary plasmids, can generate siRNAs, independently of whether these ectopic sequences can be transcribed or are promoterless. Furthermore, we show that this induction of siRNAs in agroinfiltrated tissues does not require an interaction of the T-DNA or a derivative of the T-DNA with homologous transgenic DNA or RNA sequences. We also show that, for the efficient triggering of silencing by T-DNA with homology to the $3^{\prime}$ noncoding region of a transgene, the generated siRNAs had to undergo an amplification that required the active transcription of the delivered sequences. On the other hand, the T-DNA-mediated induction of siRNAs to a transgene promoter sequence failed to induce transgene silencing.

\section{RESULTS}

Nicotiana benthamiana plants transformed with a gene encoding the green fluorescent protein (GFP) were used in the studies described below. These plants are Line $16 \mathrm{c}$ of Ruiz and associates (1998) and contain the GFP transgene flanked upstream by the cauliflower mosaic virus (CaMV) 35S RNA promoter (35SP) sequence and downstream by the nopaline synthase (Nos) transcription termination (NosT) signal. In addition, upstream of the transgene there is a neomycin phosphotransferase (nptII) gene flanked by an Nos promoter and an NosT signal, which confers antibiotic resistance.

\section{A. tumefaciens stimulates expression of a resident GFP transgene in infiltrated tissue.}

We found that infiltration of the above transgenic plants, with a suspension of $A$. tumefaciens that does not contain a binary vector, induced a steady increase in the intensity of the fluorescence of the transgene-derived GFP, with time. This increase in fluorescence was limited to the infiltrated area (Fig. 1A, left side of leaf 1). The increase in the level of fluorescence induced by infiltration with an A. tumefaciens without acetosyringone was similar to that found when acetosyringone was present (data not shown). By contrast, when leaves were infiltrated with buffer without bacteria, there was no increase of fluorescence in the infiltrated area (Fig. 1A, left side of leaf 2). Therefore, the effect of the agroinfiltration on the level of GFP-derived fluorescence is due to the bacterium itself and not to other components of the infiltration solution.

The levels of fluorescence measured by stereomicroscopy correlated with the amounts of GFP and transgene mRNA detected in infiltrated tissues. Higher levels of GFP and GFP mRNA accumulated in tissues infiltrated with A. tumefaciens harboring no binary vector than in buffer-infiltrated or noninfiltrated tissues (Fig. 2, lane 4 vs. lanes 2 and 3, and data not shown). Thus, when examining suppression of GFP expression in tissues agroinfiltrated with a binary construct containing a cassette identical to the transgene cassette, the basal level of GFP expressed from the transgene is not that observed in the surrounding noninfiltrated tissues, but is a higher level of expression caused by infiltration of the $A$. tumefaciens.

\section{A decrease in GFP fluorescence can be triggered by T-DNA sequences lacking homology \\ to the transgene coding sequences.}

When fully expanded leaves of Line 16c plants were infiltrated with $A$. tumefaciens harboring a binary plasmid containing a 35SP:GFP:NosT cassette identical to the transgene in Line 16c plants (Brigneti et al. 1998) (denoted here as $\left.\mathrm{pGFP}^{(+35,+ \text { NosT })}\right)$, the level of fluorescence in the infiltrated tissue decreased (Fig. 1A, right side of leaf 2) due to the induction of PTGS, as has been reported previously elsewhere (Voinnet et al. 1998). Different binary constructs were agroinfiltrated into leaves of Line $16 \mathrm{c}$ plants to assess the relative contributions to siRNA induction and silencing by the nontranscribed sequence in the T-DNA homologous to the transgene (the $35 \mathrm{~S}$ promoter) vs. the transcribed GFP and NosT sequences. These constructs are represented schematically in Figure 1 with different color-coded boxes indicating relevant sequence information. The presence $(+)$ or absence $(-)$ of sequence homology in the binary plasmid to the transgene $35 \mathrm{SP}$ or NosT regulatory sequences is indicated as $( \pm 35, \pm$ NosT $)$.

At 12 days post infiltration (dpi), tissues infiltrated with an A. tumefaciens carrying the plasmid $\operatorname{pGPTV}^{(-35,-N o s T)}$, which lacks any sequence homology to the 35SP:GFP:NosT cassette in the transgene, showed an increase in the level of green fluorescence, similar to that observed in tissues infiltrated with $A$. tumefaciens not harboring a binary vector (Fig. 1A, right- vs. left-infiltrated areas, respectively, in leaf 1). However, in tissues infiltrated with an A. tumefaciens containing either the empty binary plasmid pROK2 $2^{(+35,+ \text { NosT })}$, which contains $35 \mathrm{SP}$ and NosT sequences, or the binary plasmid pGPTV ${ }^{(+35,+ \text { NosT })}$ (containing a $\beta$-glucuronidase $[G U S]$ gene flanked by $35 \mathrm{SP}$ and NosT sequences), the level of GFP-derived fluorescence decreased (Fig. 1A, left-infiltrated areas in leaves 3 and 4, respectively). This loss-of-fluorescence phenotype was similar to that observed in silenced tissues due to agroinfiltration of

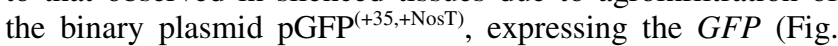
1A, compare left-infiltrated areas in leaves 3 and 4 with the right-infiltrated area in leaf 2).

Molecular analysis of the infiltrated tissues showed a strong correlation between fluorescence data and the levels of GFPspecific mRNA (Fig. 2). In tissues infiltrated with A. tumefaciens harboring either no binary vector or a vector with no sequence homology to the GFP transgene cassette pGPTV $^{(-35,- \text { NosT })}$, the high

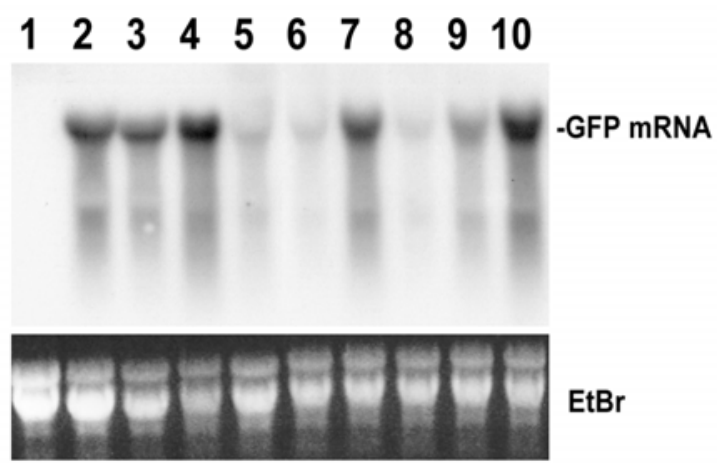

Fig. 2. Northern blot analysis of transgene mRNA accumulation in nontransformed, noninfiltrated Nicotiana benthamiana (lane 1) or $N$. benthamiana Line 16c leaves (lanes 2 to 10), noninfiltrated (lane 2) or infiltrated with buffer alone (lane 3), Agrobacterium tumefaciens harboring no vector (lane 4$)$, and A. tumefaciens harboring the binary vectors $\mathrm{pGFP}^{(+35,+ \text { NosT) }}$ (lane 5), pROK2 $2^{(+35,+ \text { NosT) }}$ (lane 6), pROK2 $2^{(+35,+ \text { NosT)}} / \mathrm{P} 1 \mathrm{HC}$ (lane 7), pGPTV $^{(+35,+ \text { NosT) }}($ lane 8$)$, pGPTV $^{(+35,+ \text { NosT })} / \mathrm{P} 1 \mathrm{HC}$ (lane 9$)$, or pGPTV ${ }^{(-35}$, -NosT) (lane 10). The lower panel shows an ethidium bromide-stained gel of the same samples as a loading control. A digoxigenin-labeled probe complementary to the green fluorescent protein-transgene mRNA was used for the hybridization analysis. 
A

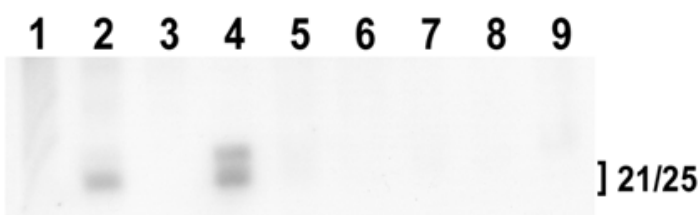

GFP Probe

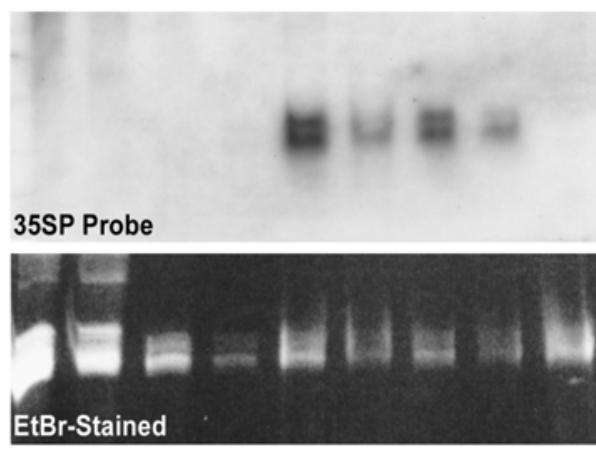

B
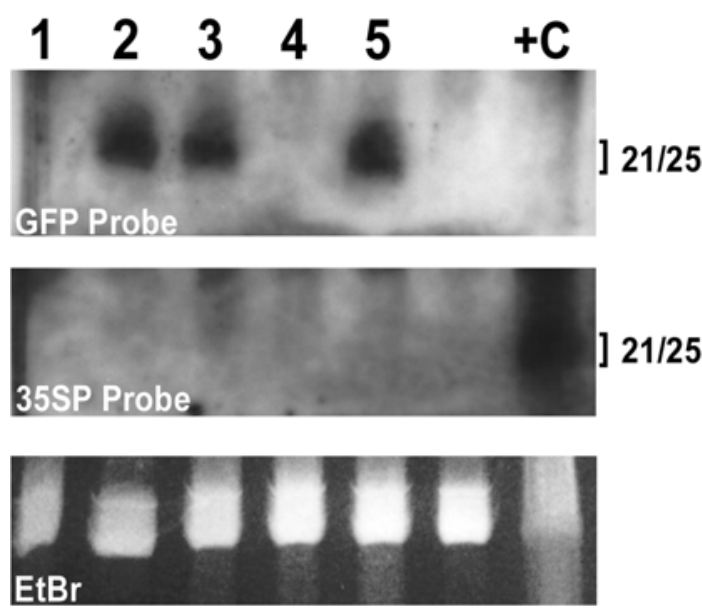

Fig. 3. A, Detection of small interfering (si)RNAs to the encoding green fluorescent protein (GFP) sequence (upper panel) and to the cauliflower mosaic virus (CaMV) 35S RNA promoter sequence (middle panel), in samples extracted from tissues of either nontransformed, noninfiltrated plants (lane 1), or GFP-transgenic Line 16c plants (lanes 2 to 9). Samples extracted from noninfiltrated tissues from a systemically silenced plant (lane 2) or from tissues infiltrated with Agrobacterium tumefaciens harboring no binary vector (lane 3 ), or plasmids pGFP ${ }^{(+35,+N o s T)}$ (lane 4$)$, pROK2 $^{(+35,+N o s T)}$ (lane 5), pROK2 $^{(+35,+ \text { NosT }) / P 1 H C ~(l a n e ~ 6), ~}$ pGPTV $^{(+35,+ \text { NosT) }}$ (lane 7), pGPTV $^{(+35,+ \text { NosT }) / P 1 H C ~(l a n e ~ 8), ~ o r ~ p G P T V ~}{ }^{(-35,- \text { NosT) }}$ (lane 9). The upper and middle panels show Northern blot hybridization analyses of the above samples against probes specific to the GFP gene (upper panel), or the CaMV 35S RNA promoter sequence (middle panel). The lower panel shows an ethidium bromide-stained gel of the same samples as a loading control. B, Analysis of the siRNA population in systemic tissue of Nicotiana benthamiana plants: Line 16c plants that had been infiltrated 3 weeks before (threeor four-leaf stage) with A. tumefaciens harboring no binary vector (lane 1), construct pGFP( ${ }^{(+35,+ \text { NosT) }}$ (lane 2), construct pROK2 $2^{(+35,+ \text { NosT) }}$ (lane 3), con-

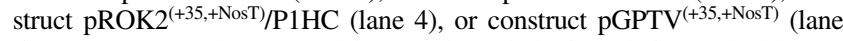
5). Lane $+\mathrm{C}$, sample extracted from a Line 16c leaf agroinfiltrated with construct pGPTV $^{(+35,+ \text { NosT })}$ as a positive control for 35SsiRNAs. Upper and middle panels show Northern blot hybridization analyses of the above samples against probes specific to the GFP gene (upper panel) or the CaMV 35S RNA promoter sequence (middle panel). The lower panel shows an ethidium bromide-stained gel of the same samples. levels of fluorescence (Fig. 1A, leaf 1) corresponded with high levels of GFP mRNA accumulation (Fig. 2, lanes 4 and 10). In tissues agroinfiltrated with plasmids $\mathrm{pROK} 2^{(+35,+ \text { NosT })}$ or

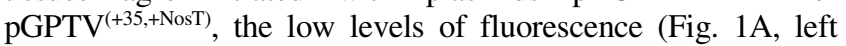
side of leaves 3 and 4, respectively) correlated with low levels of GFP mRNA accumulation (Fig. 2, lanes 6 and 8).

PTGS (Kasschau and Carrington 1998) but not TGS (Marathe et al. 2000) can be suppressed by expression of the sequences encoding the P1HCPro of potyviruses. Therefore, it was of interest to determine whether silencing triggered by these binary constructs, with sequence homology limited to the transgene promoter and transcription termination signals, could be prevented by the expression from the same binary construct of a viral suppressor of PTGS, the P1HCPro of Potato virus $Y$ (PVY). The PVY P1HCPro encoding sequence was cloned into the binary vectors $\mathrm{pROK} 2^{(+35,+ \text { NosT })}$ and pGPTV $^{(+35,+ \text { NosT) }}$ (Fig. 1A, constructs pROK2 $2^{(+35,+ \text { NosT })} / \mathrm{P} 1 \mathrm{HC}$ and pGPTV $^{(+35,+ \text { NosT }) / P 1 H C, ~ r e s p e c t i v e l y) . ~ I n ~ b o t h ~ c a s e s, ~ i n ~ t i s s u e ~}$ agroinfiltrated with these constructs, fluorescence increased relative to noninfiltrated tissues (Fig. 1A, right-infiltrated areas in leaves 3 and 4, respectively). This increase of fluorescence reached a peak at $8 \mathrm{dpi}$, after which the intensity of fluorescence in the infiltrated tissues decreased slowly (data not shown). Therefore, transient expression of the P1HCPro gene prevented the decrease in fluorescence, which otherwise would have been induced by either $\mathrm{pROK} 2^{(+35,+ \text { NosT })}$ or pGPTV $^{(+35,+ \text { NosT) }}$ (Fig. 1A, compare left- and right-infiltrated tissues in leaves 3 and 4). Moreover, the effect of the P1HCPro is limited to the areas in which it is being expressed. P1HCPro failed to prevent the silencing of the transgene in an adjacent area within the same leaf that had been agroinfiltrated with the empty vector pROK2 (Fig. 1A, compare left- and right-infiltrated areas in leaves 3 and 4). The higher levels of fluorescence that resulted from the expression in cis- of the P1HCPro (Fig. 1A, right side of leaves 3 and 4) correlated with higher levels of GFP mRNA accumulation (Fig. 2, lane 7 vs. lane 6 and lane 9 vs. lane 8 ).

\section{The relationship between active transcription of T-DNA sequences and siRNA induction in infiltrated tissue.}

We analyzed the production of siRNAs to the promoter and GFP sequences in the above agroinfiltrated tissues. In samples extracted from tissues of Line 16c plants either noninfiltrated or infiltrated with A. tumefaciens harboring no binary vectors, no siRNAs were detected by probes for the GFP coding region (Fig. 3A, upper panel, lanes 1 and 3). By contrast, in samples extracted from tissues agroinfiltrated with the plasmid $\mathrm{pGFP}^{(+35,+ \text { NosT })}$, two discrete bands corresponding to siRNAs of the GFP gene (GFPsiRNAs) were detected in approximately equal amounts (Fig. 3A, upper panel, lane 4). Moreover, in RNAs extracted from systemically silenced tissues of a Line 16c plant which had been agroinfiltrated in lower leaves with plasmid $\mathrm{pGFP}^{(+35,+ \text { NosT })}$, the lower-size RNA species accounted for most of the GFPsiRNAs detected (Fig. 3A, upper panel, lane 2). In samples extracted from tissues agroinfiltrated with binary vectors lacking the $G F P$ gene, only trace amounts of GFPsiRNAs were found (Fig. 3A, upper panel, lanes 5 to 9).

When these samples were analyzed using a probe specific to the 35SP sequence, siRNAs also were observed (35SsiRNAs) in samples extracted from tissues agroinfiltrated with binary plasmids containing 35SP sequences (Fig. 3A, middle panel, lanes 5 to 8). As was observed for the GFPsiRNAs, the 35SsiRNAs also appeared as two discrete bands of approximately the same intensity (Fig. 3A, middle panel, lanes 5 and 7 vs. upper panel, lane 4). However, in tissues in which the PlHCPro gene was expressed, the accumulation of the 35SsiRNAs was reduced, especially the accumulation of the 
larger siRNA (Fig. 3A, middle panel, lanes 6 and 8 vs. lanes 5 and 7, respectively). No 35SsiRNAs were seen in RNAs extracted from noninfiltrated tissues (Fig. 3A, middle panel, lane 1) or from tissues infiltrated with A. tumefaciens harboring either no binary vector (Fig. 3A, middle panel, lane 3) or binary vectors not containing a 35SP sequence (Fig. 3A, middle panel, lane 9). Surprisingly, only trace amounts of 35SsiRNAs were seen in RNAs extracted from plants infiltrated with A. tumefaciens harboring the plasmid $\mathrm{pGFP}^{(+35,+ \text { NosT)}}$ (Fig. 3A, middle panel, lane 4).

\section{Local silencing of the GFP gene induced by empty binary plasmids spreads systemically.}

The results shown in Figure 3A were obtained from infiltrated tissue of fully expanded leaves of Line 16c plants (as shown in Fig. 1A; plants approximately $15 \mathrm{~cm}$ high, with approximately 15 leaves). In our experience, spread of systemic silencing was greatly favored if infiltrating smaller plants. When small Line 16c plantlets (four- to six-true-leaf stage) were infiltrated with $A$. tumefaciens harboring no binary plasmid, neither local nor systemic silencing occurred after several weeks (Fig. 1B, plant 1), as in noninfiltrated plantlets (Fig. 1B, plant 6). In plantlets infiltrated with A. tumefaciens harboring plasmid $\mathrm{pGFP}^{(+35,+ \text { NosT })}$, silencing of $G F P$ initially appeared in the infiltrated tissues, and eventually spread systemically, due to PTGS (Fig. 1B, plant 2 vs. plants 1 and 6). In plantlets infiltrated with $A$. tumefaciens containing either plasmid pROK2 $2^{(+35,+ \text { NosT })}$ or pGPTV $^{(+35,+ \text { NosT })}$, systemic silencing of the GFP transgene also occurred (Fig. 1B, plants 3 and 5, respectively). By contrast, no systemic silencing occurred in plantlets agroinfiltrated with the binary plasmid $\mathrm{pROK} 2^{(+35,+ \text { NosT })} / \mathrm{P} 1 \mathrm{HC}$, which expresses the P1HCPro of PVY (Fig. 1B, plant 4).

Molecular analysis of the siRNA population from systemically silenced tissues showed that GFPsiRNAs accumulated in the above silenced plants (Fig. 3B, upper panel, lanes 2, 3, and 5 ), even in plants that had been agroinfiltrated with binary plasmids lacking the GFP gene (Fig. 3B, upper panel, lanes 3 and 5). Moreover, no 35SsiRNAs accumulated in systemically silenced tissues from any of these plants, in contrast to what was observed in tissues agroinfiltrated by the same vectors

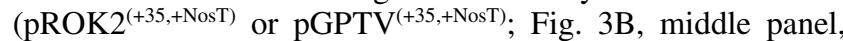
lanes 3 and 5 vs. Fig. 3A, middle panel, lanes 5 and 7). The presence of only GFPsiRNAs in the systemically silenced leaves indicates that the transgene is being silenced posttranscriptionally. In agreement with this, progeny plants, produced by self-pollination of the plants silenced by agroinfiltration of vectors pROK2 $2^{(+35,+ \text { NosT) }}$ or pGPTV ${ }^{(+35,+ \text { NosT) }}$ (Fig. 1B, plants 3 and 5), were not silenced (data not shown).

\section{Differential accumulation of siRNAs to promoter vs. transcribed sequences occurs from the same T-DNA.}

Interestingly, when the whole GFP gene was present in the T-DNA, downstream of the 35SP sequence, almost no 35SsiRNAs accumulated (Fig. 3A, middle panel, lane 4). However, the presence of the much larger GUS gene downstream of the $35 \mathrm{SP}$ sequence in construct pGPTV $^{(+35,+ \text { NosT })}$ did not prevent the accumulation of 35SsiRNAs (Fig. 3A, middle panel, lane 7 vs. lane 4). In fact, their accumulation was similar to that obtained with construct $\mathrm{pROK} 2^{(+35,+ \text { NosT })}$, which lacks the GUS sequence (Fig. 3A, middle panel, lane 7 vs. lane 5).

The above analyses showed that the induction of local and systemic silencing occurred after agroinfiltration of binary plasmids harboring 35SP and NosT sequences (pROK2 $^{(+35,+ \text { NosT) }}$ or pGPTV ${ }^{(+35,+ \text { NosT })}$; Fig. 1B, plants 3 and 5, respectively). To delineate the role played by each (the $35 \mathrm{SP}$ and the NosT sequences) in the triggering of silencing after agroinfiltration of binary plasmids, two new binary constructs were used: one containing the 35SP sequence, but lacking the

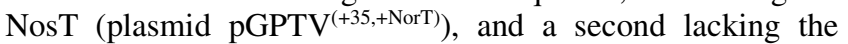
35SP sequence, but containing a promoterless NosT sequence (plasmid pGPTV ${ }^{(-35,+ \text { NosT })}$ ). These constructs are schematically represented in Figure 4. The constructs were agroinfiltrated into fully expanded leaves of Line 16c plants and the fluorescence phenotypes were monitored. In addition to the accumulation of 35SsiRNAs in infiltrated tissues, the accumulation of siRNAs to the NosT sequence (NosTsiRNAs) also was analyzed. As a control, leaves of Line 16c plants were agroinfiltrated with a plasmid containing neither the 35SP nor the NosT sequences, where the increase in fluorescence (Fig. 4, leaf 1) correlated with absence of siRNAs to the 35SP and NosT (Fig. 3A, lane 9, and data not shown).

In Line 16c leaves agroinfiltrated with plasmid pGPTV $^{(+35,}$ ${ }^{-\mathrm{NosT}}$, no silencing occurred (Fig. 4, leaf 2), despite the accumulation of 35SsiRNAs but not of NosTsiRNAs (Fig. 5A, lane 3 of upper and middle panels, respectively). This result shows that the 35SsiRNAs are not mediating the silencing of the transgene in the infiltrated tissue. However, in leaves of Line $16 \mathrm{c}$ plants agroinfiltrated with plasmid pGPTV $^{(-35,+ \text { NosT })}$, in which the NosT sequence from the ectopic DNA was not transcribed, a weak local silencing phenotype was observed (Fig. 4 , leaf 3 ), because the true basal level of fluorescence for the infiltrated area should be higher (Fig. 1A, leaf 1; Fig. 4, leaf 1). This weak silencing correlated with the absence of 35SsiRNAs and the presence of NosTsiRNAs (Fig. 5A, lane 1 of upper and middle panels, respectively). This weak local silencing did not spread systemically in small Line 16c plantlets agroinfiltrated with this plasmid (Fig. 5B, plant sample 1).

\section{Transcription of the NosT sequence}

from the T-DNA is required for systemic silencing.

In Line 16c leaves agroinfiltrated with plasmid pGPTV $^{(+35,+ \text { NosT })}$, which contains both the 35SP and NosT sequences, the local silencing (Fig. 4, leaf 4) correlated with the presence of both 35SsiRNAs, and NosTsiRNAs (Fig. 5A, lane 5 of upper and middle panels, respectively). The amount of NosTsiRNAs was much higher than that generated by construct pGPTV ${ }^{(-35,+ \text { NosT) }}$ (Fig. 5A, middle panel, lane 1). Together with the absence of any silencing induced by construct pGPTV $^{(+35,-N o s T)}$ (Fig. 4, leaf 2), these results suggest that silencing occurs after the generated NosTsiRNAs have been amplified.

The induction of systemic silencing required the 35SP sequence in addition to the NosT sequence. This may be due to the role of the 35SP sequence in promoting the transcription of the downstream NosT sequence from the T-DNA, rather than due to some promoter-targeted transcriptional silencing of the transgene mediated by the 35SsiRNAs. Thus, although the generation of the NosTsiRNAs does not require the active transcription of the ectopic NosT sequence, its amplification may require transcription. If this were the case, agroinfiltration of a binary vector, in which the NosT sequence is downstream of a constitutive promoter with little contiguous homology to the transgene promoter, would still induce strong silencing of the GFP transgene. To test this, a plasmid containing a NosT sequence downstream a promoter from the Caulimovirus, Strawberry vein banding virus (SVBV), was used in the agroinfiltration assay (plasmid $\mathrm{pGPTV}^{(+\mathrm{SVBV},+ \text { NosT) }}$, schematically represented in Fig. 4). The SVBV 35S RNA promoter has approximately $52 \%$ sequence similarity to the CaMV $35 \mathrm{~S}$ RNA promoter, with no more than 10 contiguous identical nucleotides (Wang et al. 2000). Agroinfiltration of plasmid pGPTV $^{(+\mathrm{SVBV},+ \text { NosT })}$ in leaves of Line $16 \mathrm{c}$ plants resulted in strong local silencing (Fig. 4, leaf 5), in spite of the absence of 35SsiRNAs (Fig. 5A, upper panel, lane 7), whereas NosT- 
siRNAs accumulated to levels similar to those observed in tissues infiltrated with plasmid pGPTV ${ }^{(+35,+ \text { NosT) }}$ (Fig. 5A, middle panel, lane 7 vs. 5). As in the case of agroinfiltration of plasmid pGPTV ${ }^{(+35,+ \text { NosT })}$ (Fig. 5B, plant sample 5), local silencing in Line $16 \mathrm{c}$ plants induced by agroinfiltration of plasmid pGPTV $^{(+\mathrm{SVBV},+ \text { NosT) }}$ extended systemically (Fig. 5B, plant sample 7).

\section{siRNAs are generated to T-DNA sequences}

\section{sharing no homology with the plant genome.}

In the case of promoterless, and therefore nontranscribed, TDNAs sharing sequence homology to a transgene coding region, transgene silencing was proposed to be triggered by ectopic interactions between the T-DNA and the transgene (Voinnet et al. 1998). Therefore, we wanted to determine whether the induction of siRNAs by ectopic T-DNA, especially from nontranscribed promoter sequences (35SsiRNAs), required any interaction with sequences in the plant genome homologous to those in the agroinfiltrated binary plasmid, or
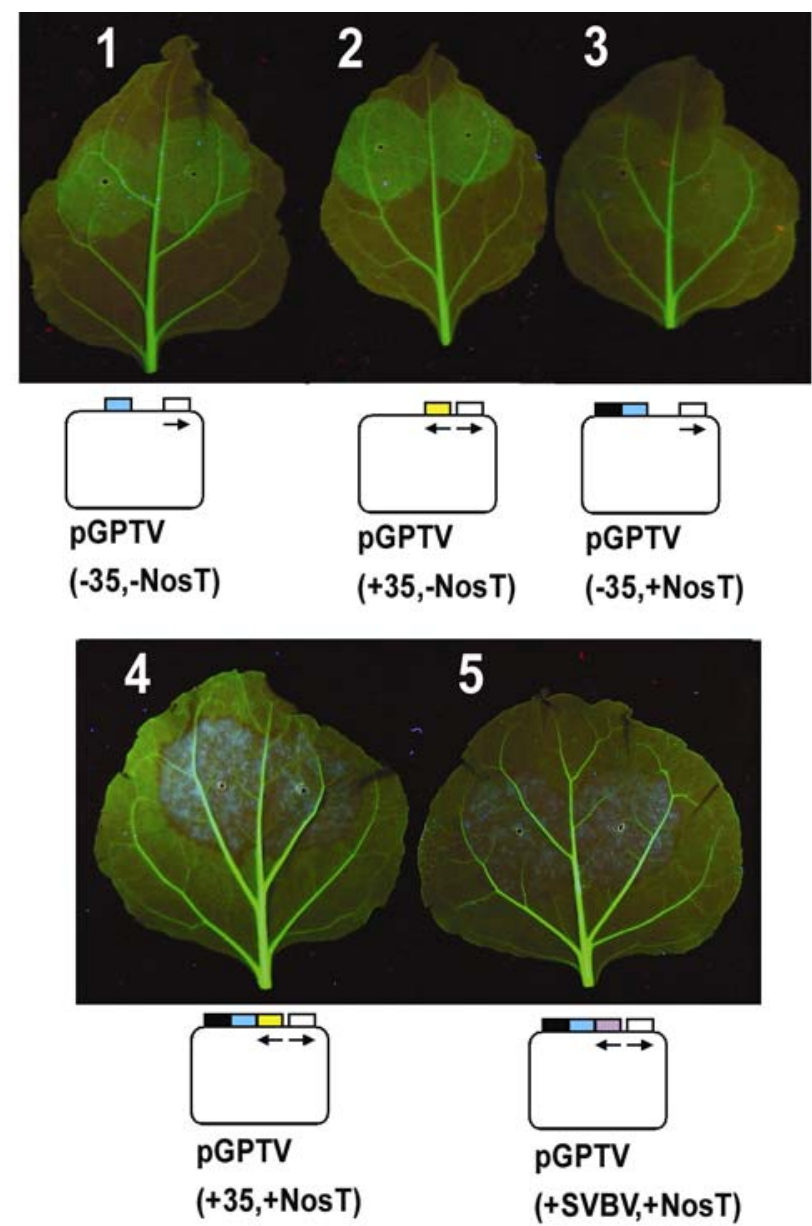

Fig. 4. UV detection of green fluorescent protein (GFP)-derived fluorescence at 12 days post infiltration of leaves from Nicotiana benthamiana Line 16c plants infiltrated with Agrobacterium tumefaciens containing the following binary vectors: pGPTV ${ }^{(-35,- \text { NosT) }}$ (leaf 1), pGPTV $^{(+35,- \text { NosT) }}$ (leaf 2), $\operatorname{pGPTV}^{(-35,+ \text { NosT) }}$ (leaf 3), $\operatorname{pGPTV}^{(+35,+ \text { NosT) }}$ (leaf 4), and pGPTV $^{(+\mathrm{SVBV},+\mathrm{NosT})}$ (leaf 5). These constructs are represented schematically under each leaf, with different color-coded rectangles indicating the different sequences: cauliflower mosaic virus (CaMV) 35SP, yellow; strawberry vein banding virus (SVBV) 35SP, violet; nopaline synthase transcription terminator signal (NosT), black; $\beta$-glucuronidase (GUS), blue; the cassette NosP:nptII:pAg7T, open rectangle. Arrows below the promoters indicate the direction of transcription. Abbreviations: 35SP, 35S RNA promoter; NosP, nopaline synthase promoter; nptII, neomycin phosphotransferase; $\mathrm{pAg} 7 \mathrm{~T}$, gene 7 poly(A) transcription termination signal. whether the ectopic DNA was itself sufficient for the triggering of siRNA accumulation. For this purpose, siRNA accumulation in leaves of Line 16c plants vs. nontransformed plants was compared after agroinfiltration with different binary plasmids containing either or both of the CaMV 35SP and the NosT sequences, or with the CaMV 35SP sequence substituted for by the SVBV 35SP sequence (Fig. 5A). Interestingly, the presence or absence of detectable siRNA accumulation in infiltrated tissues of Line $16 \mathrm{c}$ vs. nontransgenic plants was identical (Fig. 5A, lanes 1, 3, 5, and 7 vs. lanes 2, 4, 6, and 8, respectively). This indicated that the agroinfiltrated T-DNA is sufficient to trigger the generation of siRNA. Furthermore, it confirmed that the amplification of the NosTsiRNAs (with the consequent induction of silencing) did not require the transgene mRNA sequences either (Fig. 5A, middle panel, lines 5 and 7 vs. lanes 6 and 8). The presence of the transgene mRNA failed to induce detectable amplification of the NosTsiRNAs generated by nontranscribed T-DNA sequences (Fig. 5A, middle panel, lane 1 vs. 2).

\section{DISCUSSION}

In the present work, we show that agroinfiltration of a GFP transgenic plant (Line 16c; Ruiz et al. 1998) increases the level of expression of the GFP transgene in infiltrated tissue (Fig. 1A, leaf 1). This is not a trivial issue, because silencing work with these plants, which uses agroinfiltrated tissues, should take this effect into account. We also show that agroinfiltration of binary vectors with homology to the transgene limited to the noncoding regions can induce local silencing (Fig. 1A, left side of leaves 3 and 4) that eventually extends systemically under the appropriate conditions (Fig. 1B, plants 3 and 5). A practical consequence of this observation is that the Line $16 \mathrm{c}$ GFP transgene, and perhaps other transgenes, can be silenced by using empty binary transformation vectors (such as pROK2). Furthermore, our system provides a simple approach for the study of putative viral suppressors of gene silencing (Voinnet et al. 1999). By inserting the sequences of putative suppressors in binary vectors, flanked between promoter and transcription termination sequences homologous to the Line $16 \mathrm{c}$ transgene, it is possible to address whether their transient expression in cis-, in agroinfiltrated tissues, prevents or delays the elicitation of gene silencing mediated by the T-DNA sequences homologous to the transgene $3^{\prime}$ noncoding regions, as we have shown here for the PVY P1HCPro (Fig. 1A, right side of leaves 3 and 4; Fig. 3A, lanes 6 and 8 vs. 5 and 7).

Our results show that the T-DNA agroinfiltrated into plants can initiate the production of siRNAs in infiltrated tissues, corresponding to the sequences present in the binary DNA (35SP, GFP, or NosT sequences analyzed here; Figs. 3 and 5A). This occurs even in the case of sequences that are not actively transcribed from the ectopic DNA (the 35SP sequence in all the constructs, or the NosT sequence in construct PGPTV $\left.^{(-35,+ \text { NosT })}\right)$. Triggering of gene silencing by promoterless T-DNA sequences had been reported previously after introduction of sequences homologous to the transgene coding region, requiring an interaction between the ectopic DNA and the transgene nucleic acid (Voinnet et al. 1998). We have found that the induction of siRNAs to nonactively transcribed T-DNA sequences in infiltrated tissues occurs in nontransformed plants and, therefore, does not require an interaction of the ectopic sequences with homologous DNA or RNA sequences from the plant (Fig. 5A). Moreover, we also found that the presence of siRNAs to nontranscribed sequences from the T-DNA, whether to 35SsiRNAs (Fig. 5A, lane 3) or to NosTsiRNAs (Fig. 5A, lane 1) did not in itself provoke systemic silencing (Fig. 5B, plant samples 3 and 1, respectively). 
How do these siRNAs triggered by T-DNA originate? Delivery of T-DNA into the plant cell by $A$. tumefaciens takes place as a linear, ssDNA. This T-DNA contains the R border at its $5^{\prime}$ end and the L border at its $3^{\prime}$ end (Tinland et al. 1994). The strand that enters the cell in the case of pROK2 is the sense strand; whereas, in the case of the pGPTV constructs, it is the antisense strand. Once inside the cell, ssDNA can be transformed rapidly into a dsDNA form, as shown by observations that the transient rate of expression of genes from ectopic ssDNA in either orientation is similar (Rodenburg et al. 1989). In this regard, agroinfiltration of either type of construct induced silencing equally (Fig. 1A, left side of leaves 3 and 4; Fig. 1B, plants 3 and 5).

Cryptic transcription of $35 \mathrm{SP}$ sequences from the Nos promoter is theoretically possible in pROK2, and in the promoterless GFP-expressing binary vector used by Voinnet and associates (1998). However, this is not possible in linear T-DNA derived from pGPTV constructs: the pGPTV constructs also differ from pROK2 in that the selectable marker gene nptII, which confers antibiotic resistance (under the control of the Nos promoter), is orientated in a direction opposite to that of the 35SP (Fig. 1A). This eliminates cryptic transcription of $35 \mathrm{SP}$ sequences occurring by readthrough of the termination signal downstream of the nptII gene.

After the T-DNA sequences integrate into the plant genome, cryptic transcription of such T-DNA sequences may occur from a plant promoter upstream or downstream of the T-DNA. This would result in ssRNA corresponding to the 35SP sequences or the NosT sequences, respectively. Such ssRNA, representing products of aberrant transcription, may be converted to dsRNAs by the plant RNA-dependent RNA polymerases (RdRp) (Dalmay et al. 2000; Mourrain et al. 2000) and then be degraded to the siRNA. Alternatively, plant RdRps are known to accept ssDNA as templates for RNA synthesis (Schiebel et al. 1993); thus, the ssT-DNA derived from the binary DNA may be the direct template for generation of precursors of the siRNAs. Nevertheless, in the case of the siRNAs generated against the 35SP by either approach, these siRNAs in themselves did not lead to silencing of the promoter or the adjacent (GFP) coding sequences (Fig. 4, leaf 2; Fig. 5A, lane 3 , and B, plant sample 3).

How does the generation of these siRNAs corresponding to transgene noncoding regions relate to the silencing of the transgene? The presence in infiltrated tissues of NosTsiRNAs initiated by promoterless, T-DNA sequences induced a weak, delayed silencing, limited to the infiltrated areas (Fig. 4, leaf 3; Fig. 5B, plant sample 1). On the other hand, NosTsiRNAs accumulation, initiated by transcribed sequences from the $\mathrm{T}$ DNA, induced strong local and systemic silencing (Fig. 4, leaves 4 and 5; Fig. 5B, plant samples 5 and 7). In the latter examples, the accumulation of NosTsiRNAs in infiltrated tissues was higher than in the former situation (Fig. 5A, lanes 5 and 7 vs. lane 1). This observation suggests that there is a relationship between higher accumulation of NosTsiRNAs and efficient silencing, and that higher accumulation is achieved from T-DNA sequences that are actively transcribed. Transgene silencing by tobacco rattle virus vectors carrying NosT sequences, in which these RNA sequences are actively replicated, has been reported (Vaistij et al. 2002).

The presence of GFPsiRNAs in systemically silenced tissues is explained by the progression $3^{\prime} \rightarrow 5^{\prime}$ on the transgene mRNA of siRNA production (Lipardi et al. 2001; Sijen et al. 2001). However, this posttranscriptional NosT-mediated silencing does not appear to extend to some other organisms (Galvani and Sperling 2001). Our results also are in agreement with observations made by others using Drosophila embryo extracts. In that case, RNAi did not occur below a threshold of
siRNA concentration (Lipardi et al. 2001). Moreover, amplification of siRNA accumulation in plants required the interaction with transcript RNAs in a primer or template mode, as also was shown for RNAi (Lipardi et al. 2001). It is not clear
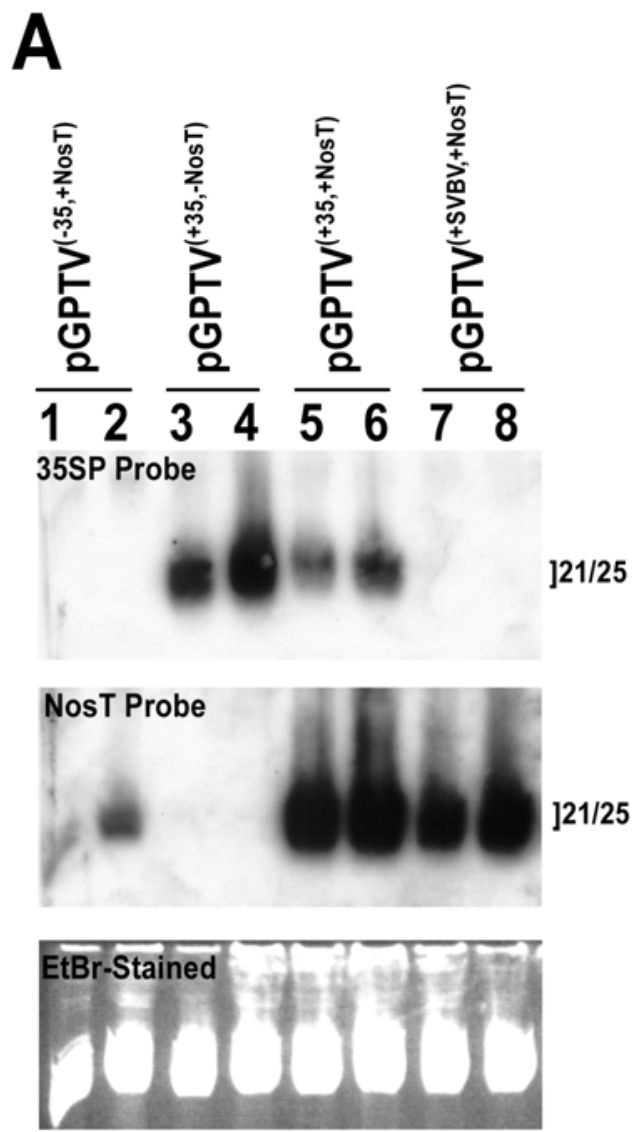

B

\section{Plant samples}

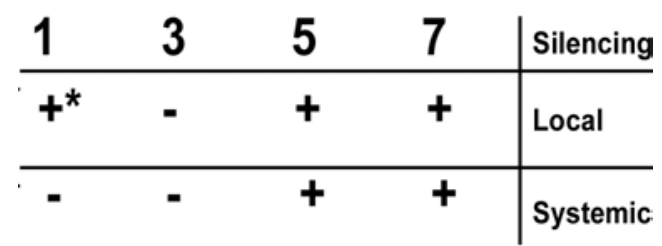

Fig. 5. A, Northern blot hybridization analysis of the siRNA population to the cauliflower mosaic virus (CaMV) 35S RNA promoter and the nopaline synthase transcription termination (NosT) signal in tissue of nontransgenic Nicotiana benthamiana (lanes 2, 4, 6, and 8) or Line 16c green fluorescent protein (GFP)-transgenic plants (lanes 1, 3, 5, and 7) infiltrated with Agrobacterium tumefaciens containing the following binary vectors: pGPTV $^{(-35,+ \text { NosT) }}$ (lanes 1 and 2), pGPTV ${ }^{(+35,- \text { NosT) }}$ (lanes 3 and 4), pGPTV $^{(+35,+ \text { NosT) }}\left(\right.$ lanes 5 and 6), and pGPTV ${ }^{(+\mathrm{SVBV},+ \text { NosT) }}($ lanes 7 and 8). Northern blot hybridization analyses of the above samples against probes specific to the CaMV 35S RNA promoter (upper panel), or the NosT signal (middle panel). The lower panel shows an ethidium bromidestained gel of the same samples as a control for equal loading. B, Presence (+) or absence (-) of induced local or systemic silencing is indicated, following agroinfiltration of Line 16c plants with the constructs shown in A: pGPTV ${ }^{(-35,+ \text { NosT) }}$ (lane 1; the asterisk indicates that the silencing is weak), pGPTV ${ }^{(+35,- \text { NosT) }}$ (lane 3), pGPTV ${ }^{(+35,+ \text { NosT) }}$ (lane 5), and pGPTV $^{(+\mathrm{SVBV},+ \text { NosT) }}$ (lane 7). Local silencing was monitored in infiltrated fully expanded leaves. Systemic silencing was monitored in infiltrated small plantlets (four- to six-true-leaf stage). The plant sample numbers correspond to those plants used to analyze siRNA production in A. 
why amplification of NosTsiRNAs did not occur in the presence of the transgene mRNA (Fig. 5A, middle panel, lane 1 vs. lane 2), whereas it occurred in the presence of NosT transcripts derived from the ectopic DNA (Fig. 5A, middle panel, lanes 5 to 8 vs. lanes 1 and 2). In contrast to the failure of promoterless ectopic NosT sequences to trigger efficient transgene silencing, promoterless ectopic DNA sequences with homology to the transgene coding region efficiently triggered silencing (Voinnet et al. 1998). It may be possible that the $3^{\prime}$ noncoding region of the transgene mRNA is less accessible to the silencing degradation machinery than the same region in the transcript derived from the ectopic DNA. In this regard, NosT-mediated silencing was not detected between single copy transgenes in plants (Charrier et al. 2000).

NosT-mediated local and systemic silencing was achieved in the absence of 35SsiRNAs, using a different promoter (Fig. 4, leaf 5; Fig. 5A, upper panel, lane 7, and B, plant sample 7). This demonstrated further that silencing of the transgene did not require the $35 \mathrm{SsiRNAs}$. Moreover, this was in agreement with the observation that infiltration of a binary construct with sequence homology limited to the transgene 35SP failed to trigger any silencing (Fig. 4, leaf 2; Fig. 5B, plant sample 3), despite the presence of 35SsiRNAs (Fig. 5A, upper panel, lane $3)$. However, we cannot rule out that, in other systems, the 35SsiRNAs play an additional role in promoter silencing leading to TGS. Transgene promoter methylation, directed by dsRNA and siRNAs, has been reported (Mette et al. 1999, 2000). In our system, the generation of only 35SsiRNAs in infiltrated tissues failed to induce silencing (Fig. 4, leaf 2; Fig. 5A, upper panel, lane 3 and B, plant sample 3 ). This may be related to the lack of amplification of the 35SsiRNAs, because there is no transcription of the transgene 35SP. Perhaps these 35SsiRNAs are able to trigger some RNA-directed DNA methylation of the transgene promoter, insufficient to trigger a noticeable transcriptional silencing of the transgene in the infiltrated tissue, during the time frame of the experiment. Previous experimental work using agroinfiltration techniques concluded that promoter sequences in the ectopic DNA not transcribed into RNA played no role in transgene silencing (Schöb et al. 1997; Voinnet et al. 1998). This is consistent with our observation of the differential accumulation of siRNAs in infiltrated tissues of Line 16c plants (i.e., very little 35SsiRNAs accumulated when GFPsiRNAs accumulated; Fig. 3A, lane 4).

\section{MATERIALS AND METHODS}

\section{Plants and Agrobacterium strains.}

Nontransformed plants as well as $N$. benthamiana plants transformed with a single copy of a 35SP:GFP:NosT transgene (Line 16c; Ruiz et al. 1998) were used in the agroinfiltration experiments. Prior to and after agroinfiltration, plants were kept in a growth chamber at $25^{\circ} \mathrm{C}$, with a 16 -h daylight period. A. tumefaciens strains LBA-4404 and C58C1, were both used for the agroinfiltration studies with similar results. All the experimental data shown in the paper was generated using $A$. tumefaciens strain $\mathrm{C} 58 \mathrm{C} 1$, and the results shown in Figures 1 and 2 were reproduced using strain LBA 4404.

\section{Binary constructs.}

For convenience, in all the binary vectors used in this article, the presence or absence of the 35SP or the NosT sequences has been indicated in superscript. Thus, construct pROK $2^{(+35,+N o s T)}$ refers to the binary vector pROK2, which contains a CaMV 35S RNA promoter and a Nos transcription termination signal. Construct $\mathrm{pGPTV}^{(-35,+ \text { NosT })}$ refers to construct pGPTV-Kan (Becker et al. 1992), which contains a promoterless GUS gene and a Nos transcription termination sig- nal. Construct pGPTV ${ }^{(+35,+ \text { NosT })}$ is a derivative of pGPTV-Kan, containing a CaMV 35S RNA promoter inserted between the $S a l \mathrm{I}$ and $\mathrm{X} b a \mathrm{I}$ restriction sites (N. Wood, unpublished data). In order to clone the P1HCPro-encoding sequence of PVY into pROK2 $2^{(+35,+ \text { NosT })}$ and pGPTV $^{(+35,+ \text { NosT })}$, the corresponding sequence was amplified by the reverse-transcriptase polymerase chain reaction (RT-PCR) from an RNA preparation of a PVY-O isolate from the Scottish Crop Research Institute (SCRI) collection. For this purpose, upstream primers homologous to nucleotides $190-210$ of the PVY sequence (5'ATGGCAACTTACACATCAAC- $3^{\prime}$ ), with added BamHI or NheI $5^{\prime}$ terminal sequences, were used in conjunction with downstream primers complementary to nucleotides 2,390 2,404 of the PVY sequence (5'-CATTATAGAGTTGGT-3'), preceded by a translation termination codon (TAG) and SacI or SmaI sequences at the $5^{\prime}$ end. The amplified PCR product then either was digested with BamHI and SacI and cloned into pROK2 linearized with the same enzymes, or digested with SmaI and NheI and cloned into pGPTV $^{(+35,+ \text { NosT })}$, linearized with $X b a \mathrm{I}$ and SmaI. All constructs were multiplied in Escherichia coli strain DH5 $\alpha$ cells, under kanamycin selection, prior to their electroporation into cells of A. tumefaciens. Construct $\mathrm{pGFP}^{(+35,+ \text { NosT })}$ refers to a binary vector containing a 35SP:GFP:NosT cassette identical to the transgene of Line 16c (Brigneti et al. 1998). Construct pGPTV ${ }^{(-35,- \text { NosT) }}$ was obtained from construct pGPTV $^{(-35,+ \text { NosT) }}$ after digestion with EcoRI and SacI, and replacement of the DNA fragment released with a DNA fragment from the polylinker of pUC18, digested with the same two restriction enzymes. Construct pGPTV $^{(+35,-N o s T)}$ was obtained by digesting construct pGPTV $^{(+35,+ \text { NosT })}$ with $\mathrm{XbaI}$ and HindIII, and cloning the DNA fragment containing the $35 \mathrm{SP}$ sequence into construct $\mathrm{pGPTV}^{(-35,- \text { NosT })}$, previously linearized with $X b a \mathrm{I}$ and HindIII. Schematic representations of each binary vector are shown in Figures 2 and 5. Construct $\mathrm{pGPTV}^{(+\mathrm{SVBV},+\mathrm{NosT})}$ is a derivative of pGPTV-Kan, containing an inserted SVBV 35S RNA promoter (Wang et al. 2000; N. Wood, unpublished data).

\section{Agroinfiltration procedure and detection of GFP fluorescence.}

The agroinfiltration technique used in this work has been described before (English et al. 1997; Voinnet et al. 1998). Briefly, A. tumefaciens cultures were grown overnight in Luria broth medium containing antibiotic selection, $10 \mathrm{mM}$ MES (morpholineethanesulfonic acid), and $20 \mu \mathrm{M}$ acetosyringone. The cells were harvested by centrifugation and were resuspended in infiltration buffer: $10 \mathrm{mM} \mathrm{MgCl} 2,10 \mathrm{mM} \mathrm{MES}$, and $150 \mu \mathrm{M}$ acetosyringone. This suspension was infiltrated into $N$. benthamiana leaves by gently pressing the tip of a 2-ml disposable syringe, without needle, on the leaf surface. The resulting infiltrated tissue had a circular shape, with an average diameter of approximately $2 \mathrm{~cm}$, in fully expanded leaves. Usually two loci were infiltrated per leaf, two leaves per plant, and two plants per sample, in each experiment. This yielded a total of eight infiltrated circular regions per sample in each experiment. Each experiment was performed at least four times. After infiltration, the level of GFP-derived fluorescence, inside and outside the infiltrated region, was monitored in situ with the aid of a Leica MZ FLIII fluorescence stereo microscope, equipped with a fluorescence GFP1 filter (excitation filter 425/60 nm, barrier filter $480 \mathrm{~nm}$; Leica, Heerbrugg, Switzerland) coupled to a digital video camera (Model KY-F55B; Photonic Science, Milham, U.K.). Quantification of green fluorescence was made using Image-Pro Plus software (Media Cybernetics, Silver Spring, MD, U.S.A.). In some experiments, measures were taken from nondetached leaves, at 4-day intervals, in order to obtain time course data on the evolution 
of fluorescence (Fig. 1A). In other experiments, the degree of fluorescence was measured at 12 dpi from freshly detached leaves (Figs. 1A and 4) prior to the extraction of nucleic acid and protein for further analysis.

Imaging of GFP fluorescence in whole leaves and plants (Figs. 1 and 4) was obtained with an Olympus CAMEDIA E10 digital camera (Olympus Optical Co., Tokyo), under illumination using a black ray long-wave ultraviolet lamp (model B, 100 Amp, UV Products, Upland, CA, U.S.A.). Monitoring for systemic silencing in small plants agroinfiltrated at the 6- to 8leaf stage was performed at 3 weeks post infiltration with the aid of the ultraviolet lamp. All digital images were processed with Adobe Photoshop 6.0 software (Adobe Systems, San Jose, CA, U.S.A.).

\section{Nucleic acid and protein analysis.}

For protein and mRNA content analysis, samples containing 1 $\mathrm{g}$ of plant tissue were ground to a powder in liquid nitrogen in a mortar and were resuspended in $1 \mathrm{ml}$ of extraction buffer (100 $\mathrm{mM}$ Tris $\mathrm{HCl}, \mathrm{pH} 8,10 \mathrm{mM}$ EDTA, $1 \%$ sodium dodecyl sulfate, $0.1 \mathrm{M} \mathrm{LiCl})$. An aliquot $(100 \mu \mathrm{l})$ of the suspended sample was taken for protein analysis. The remaining sample was treated as described by Verwoerd and associates (1989) to extract total nucleic acids. RNA was precipitated from the total nucleic acid extracts by overnight incubating on ice, in the presence of $2 \mathrm{M}$ $\mathrm{LiCl}$. The precipitated RNA was resuspended in $500 \mu \mathrm{l}$ of water. RNA samples $(5 \mu \mathrm{l})$ were fractionated by electrophoresis under denaturing conditions ( $6 \%$ formaldehyde), blotted to nitrocellulose membranes, and hybridized to digoxigenin-labeled RNA probes against the GFP gene. A probe complementary to the whole GFP gene was obtained from clone TU\#65 (Chalfie et al. 1994), after linearization with KpnI, using T7 RNA polymerase. Synthesis of the probe and processing of the blots with digoxigenin-labeled probes were performed according to the manufacturer's instructions (Roche Diagnostics, Lewes, U.K.).

Low molecular weight RNAs were isolated, fractionated in polyacrylamide gels, and electroblotted as described by Llave and associates (2000). For the detection of GFPsiRNAs, a radioactive RNA probe was obtained from clone Tu\#65 (Chalfie et al. 1994) as a described above, using $\left[{ }^{32} \mathrm{P}\right]-\alpha-U T P$. To detect 35 SsiRNAs, a DNA fragment containing the $35 \mathrm{SP}$ sequence was amplified by the PCR from construct $\mathrm{pGFP}^{(+35,+ \text { NosT })}$ using a $5^{\prime}$ primer homologous to the pBI121 polylinker sequence $5^{\prime}$ of the start of the 35SP sequence (AAGCTTGCATGCCTGCAG), in conjunction with a $3^{\prime}$ end primer complementary to the first 22 nucleotides corresponding to the ER signal peptide at the $\mathrm{N}$-terminus of the transgenic GFP (ATGAAGACTAATCTTTTTCTCT) (Haseloff et al. 1997). The PCR product was digested with Pst I and $X b a \mathrm{I}$ and cloned into pSK (to produce clone pSK35SP). A radioactive probe complementary to the whole 35SP sequence was obtained by linearization of this clone with PstI and using T3 RNA polymerase. For the detection of NosTsiRNAs, the Nos transcription termination signal sequence was excised from pROK2 by digestion with $S a c \mathrm{I}$ and $E c o$ RI, and cloned into pSK linearized with the same enzymes, to produce clone pSKNosT. A radioactive probe complementary to the whole NosT sequence was obtained by linearization of this clone with SacI and using T3 RNA polymerase.

Samples for protein analysis were fractionated by sodium dodecyl sulfate-polyacrylamide gel electrophoresis $(12.5 \%$ acrylamide gels), electroblotted to PVDF Sequi-Blot membranes (Bio-Rad, Hercules, CA, U.S.A.) as described by Towbin and associates (1979), and probed with either a polyclonal antiserum against GFP (Molecular Probes, Eugene, OR, U.S.A.) or, in order to detect transient expression, monoclonal antibody 1A11 against PVY HCPro (Canto et al. 1995).

\section{ACKNOWLEDGMENTS}

This work was supported by a grant-in-aid from the Scottish Executive Environment and Rural Affairs Department, and F. Cillo was supported by a Marie Curie fellowship (HPMF-CT2000-00632) of the European Commission Programme "Human Potential". We thank D. Baulcombe and O. Voinnet from the Sainsbury Laboratory, Norwich, U.K., for providing transgenic $N$. benthamiana plants, Line 16c, A. tumefaciens strain C58C1, and the binary vector pGFP ${ }^{(+35,+ \text { NosT)}}$; and N. Wood, from SCRI, Dundee, U.K., for providing the binary vectors $\operatorname{pGPTV}^{(+35,+ \text { NosT })}$ and pGPTV $^{(+S V B V,+N o s T)}$

\section{LITERATURE CITED}

Becker, D., Kemper, E., Schell, J., and Masterson, R. 1992. New plant binary vectors with selectable markers located proximal to the left TDNA border. Plant Mol. Biol. 20:1195-1197.

Bernstein, E., Caudy, A. A., Hammond, S. M., and Hannon, G. J. 2001. Role for a bidentate ribonuclease in the initiation step of RNA interference. Nature 409:363-366.

Brigneti, G., Voinnet, O., Li, W.-X., Ji, L.-H., Ding, S.-W., and Baulcombe, D. C. 1998. Viral pathogenicity determinants are suppressors of transgene silencing in Nicotiana benthamiana. EMBO (Eur. Mol. Biol. Organ.) J. 17:6739-6746.

Canto, T., Ellis, P., Bowler, G., and López-Abella, D. 1995. Production of monoclonal antibodies to potato virus $\mathrm{Y}$ helper component-protease and their use for strain differentiation. Plant Dis. 79:234-237.

Chalfie, M., Tu, Y., Euskirchen, G., Ward, W. W., and Prasher, D. C. 1994 Green fluorescent protein as a marker for gene expression. Science 263:802-804.

Charrier, B., Scollan, C., Ross, S., Zubko, E., and Meyer, P. 2000. Cosilencing of homologous transgenes in tobacco. Mol. Breed. 6:407-419.

Cogoni, C., and Macino, G. 1999. Gene silencing in Neurospora crassa requires a protein homologous to RNA-dependent RNA polymerase. Nature 399:166-169.

Dalmay, T., Hamilton, A., Rudd, S., Angell, S., and Baulcombe, D. C. 2000. An RNA-dependent RNA polymerase gene in Arabidopsis is required for posttranscriptional gene silencing mediated by a transgene but not by a virus. Cell 101:543-553.

Elbashir, S. M., Lendeckel, W., and Tuschl, T. 2001. RNA interference is mediated by 21- and 22-nucleotide RNAs. Genes Dev. 15:188-200

English, J. J., Davenport, G. F., Elmayan, T., Vaucheret, H., and Baulcombe, D. C. 1997. Requirement of sense transcription for homology-dependent virus resistance and trans-inactivation. Plant J. 12:597603.

Fagard, M., Boutet, S., Morel, J., Bellini, C., and Vaucheret, H. 2000. AGO1, QDE-2, and RDE-1 are related proteins required for post-transcriptional gene silencing in plants, quelling in fungi, and RNA interference in animals. Proc. Natl. Acad. Sci. U.S.A. 97:11650-11654.

Galvani, A., and Sperling, L. 2001. Transgene-mediated post-transcriptional gene silencing is inhibited by $3^{\prime}$ non-coding sequences in Paramecium. Nucleic Acids Res. 29:4387-4394.

Grishok, A., Pasquinelli, A. E., Conte, D., Li, N., Parrish, S., Ha, I. Baillie, D. L., Fire, A., Ruvkun, G., and Mello, C. C. 2001. Genes and mechanisms related to RNA interference regulate expression of the small temporal RNAs that control $C$. elegans developmental timing. Cell 106:23-34

Hamilton, A. J., and Baulcombe, D. C. 1999. A species of small antisense RNA in posttranscriptional gene silencing in plants. Science 286:950951.

Hammond, S. M., Bernstein, E., Beach, D., and Hannon, G. J. 2000. An RNA-directed nuclease mediates post-transcriptional gene silencing in Drosophila cells. Nature 404:293-296.

Haseloff, J., Siemering, K. R., Prashner, D. C., and Hodge, S. 1997. Removal of a cryptic intron and subcellular localization of green fluorescent protein are required to mark transgenic Arabidopsis plants brightly. Proc. Natl. Acad. Sci. U.S.A. 94:2122-2127.

Johansen L. K., and Carrington, J. C. 2001. Silencing on the spot. Induction and suppression of RNA silencing in the Agrobacterium-mediated transient expression system. Plant Physiol. 126:930-938.

Jones, A. L., Thomas, C. L., and Maule, A. J. 1998. De novo methylation and co-suppression induced by a cytoplasmically replicating plant RNA virus. EMBO (Eur. Mol. Biol. Organ.) J. 17:6385-6393.

Jones, L., Hamilton, A. J., Voinnet, O., Thomas, C. L., and Maule, A. J. 1999. RNA-DNA interactions and DNA methylation in post-transcriptional gene silencing. Plant Cell 11:2291-2301.

Kasschau, K. D., and Carrington J. C. 1998. A counterdefensive strategy of plant viruses: Suppression of posttranscriptional gene silencing. Cell 95:461-470. 
Lagos-Quintana, M., Rauhut, R., Lendeckel, W., and Tuschl, T. 2001. Identification of novel genes coding for small expressed RNAs. Science 294:853-858

Lau, N. C., Lim, L. P., Weinstein, E. G., and Bartel, D. P. 2001. An abundant class of tiny RNAs with probable regulatory roles in Caenorhabditis elegans. Science 294:858-862.

Lee, R. C., and Ambros, V. 2001. An extensive class of small RNAs in Caenorhabditis elegans. Science 294:862-864.

Lipardi, C., Wei, Q., and Paterson, B. M. 2001. RNAi as random degradative PCR: siRNA primers convert mRNA into dsRNAs that are degraded to generate new siRNAs. Cell 107:297-307.

Llave, C., Kasscchau, K. D., and Carrington, J. C. 2000. Virus-encoded suppressor of posttranscriptional gene silencing targets a maintenance step in the silencing pathway. Proc. Natl. Acad. Sci. U.S.A. 97:13401-13406.

Marathe, R., Smith, T. H., Anandalakshimi, R., Bowman, L. H., Fagard, M., Mourrain, P., Vaucheret, H., and Vance, V. B. 2000. Plant viral suppressors of post-transcriptional silencing do not suppress transcriptional silencing. Plant J. 22:51-54.

Mette, M. F., Aufsatz, W., van der Winden, J., Matzke, M. A., and Matzke, A. J. M. 2000. Transcriptional silencing and promoter methylation triggered by double-stranded RNA. EMBO (Eur. Mol. Biol. Organ.) J. 19:5194-5201.

Mette, M. F., van der Winden, J., Matzke, M. A., and Matzke, A. J. M. 1999. Production of aberrant promoter transcripts contributes to methylation and silencing of unlinked homologous promoters in trans. EMBO (Eur. Mol. Biol. Organ.) J. 18:241-248.

Mourrain, P., Béclin, C., Elmayan, T., Feuerbach, F., Godon, C., Morel, J., Jouette, D., Lacombe, A., Nikic, S., Picault, N., Rémoué, K., Sanial, M., Vo, T., and Vaucheret, H. 2000. Arabidopsis SGS2 and SGS3 genes are required for posttranscriptional gene silencing and natural virus resistance. Cell 101:533-542.

Nykänen, A., Haley, B., and Zamore, P. D. 2001. ATP requirements and small interfering RNA structure in the RNA interference pathway. Cell 107:309-321.

Pélissier, T., Thalmeir, S., Kempe, D., Sänger, H. L., and Wassenegger, M. 1999. Heavy de novo methylation at symmetrical and non-symmetrical sites is a hallmark of RNA-directed DNA methylation. Nucleic Acids Res. 27:1625-1634.

Ratcliff, F., Harrison, B. D., and Baulcombe, D. C. 1997. A similarity between viral defense and gene silencing in plants. Science 276:15581560 .

Rodenburg, K. W., de Groot, M. J. A., Schilperoot, R. A., and Hooykaas, P. J. J. 1989. Single-stranded DNA used as an efficient new vehicle for transformation of plant protoplasts. Plant Mol. Biol. 13:711-719.

Ruiz, M. T., Voinnet, O., and Baulcombe, D. C. 1998. Initiation and maintenance of virus-induced gene silencing. Plant Cell 10:937-946.

Schiebel, W., Haas, B., Marinković, S., Klanner, A., and Sänger, H. L. 1993. RNA-directed RNA polymerase from tobacco leaves. J. Biol. Chem. 263:11851-11867
Schöb, H., Kunz, C., and Meins, F. 1997. Silencing of transgenes introduced into leaves by agroinfiltration: A simple, rapid method for investigating sequence requirements for gene silencing. Mol. Gen. Genet. 256:581-585

Sijen, T., Fleenor, J., Simmer, F., Thijssen, K. L., Parrish, S., Timmons, L., Plasterk, R. H. A., and Fire, A. 2001. On the role of RNA amplification in dsRNA-triggered gene silencing. Cell 107:465-476.

Thomas, C. L., Jones, L., Baulcombe, D. C., and Maule, A. J. 2001. Size constrains for targeting post-transcriptional gene silencing and for RNA-directed methylation in Nicotiana benthamiana plants using a potato virus $X$ vector. Plant J. 25:417-425.

Tinland, B., Hohn, B., and Puchta, H. 1994. Agrobacetrium tumefaciens transfers single-stranded transferred DNA (T-DNA) into the plant cell nucleus. Proc. Natl. Acad. Sci. U.S.A. 91:8000-8004.

Towbin, H., Staehlin, T., and Gordon, J. 1979. Electrophoretic transfer of proteins from polyacrylamide gels to nitrocellulose sheets: Procedure and some applications. Proc. Natl. Acad. Sci. U.S.A. 76:4350-4354.

Tuschl, T. 2001. RNA interference and small interfering RNAs. Chembiochem 2:239-245.

Vaistij, F. E., Jones, L., and Baulcombe, D. C. 2002. Spreading of RNA targeting and DNA methylation in RNA silencing requires transcription of the target gene and a putative RNA-dependent RNA polymerase. Plant Cell 14:857-867.

Vaucheret, H., and Fagard, M. 2001. Transcriptional gene silencing in plants: Targets, inducers and regulators. Trends Genet. 17:29-35.

Verwoerd, T. C., Dekker, B. M. M., and Hoekema, A. 1989. A small-scale procedure for the rapid isolation of plant RNAs. Nucleic Acids Res. 17:2362.

Voinnet, O. 2001. RNA silencing as a plant immune system against viruses. Trends Genet. 17:449-459.

Voinnet, O., Pinto, Y. M., and Baulcombe, D. C. 1999. Suppression of gene silencing: A general strategy used by diverse DNA and RNA viruses of plants. Proc. Natl. Acad. Sci. U.S.A. 96:14147-14152

Voinnet, O., Vain, P., Angell, S., and Baulcome, D. C. 1998. Systemic spread of sequence-specific transgene RNA degradation in plants is initiated by localized introduction of ectopic promoterless DNA. Cell 95:177-187.

Wang, Y., Gaba, V., Wolf, D., Xia, X. D., Zelcer, A., and Gal-On, A. 2000. Identification of a novel plant virus promoter using a potyvirus infectious clone. Virus Genes 20:11-17.

Wassenegger, M., Heimes, S., Riedel, L., and Sänger, H. L. 1994. RNAdirected de novo methylation of genomic sequences in plants. Cell 76:567-576.

Wightman, B., Ha, I., and Ruvkun, G. 1993. Postranscriptional regulation of the heterochronic gene lin-14 by lin- 4 mediates temporal pattern formation in C. elegans. Cell 75:855-862.

Zamore, P. D., Tuschl, T., Sharp, P. A., and Bartel, D. P. 2000. RNAi: Double-stranded RNA directs the ATP-dependent cleavage of mRNA at 21 to 23 nucleotide intervals. Cell 101:25-33. 